\title{
Fortalecimiento de la Identidad Cultural desde la investigación como estrategia Pedagógica ${ }^{1}$
}

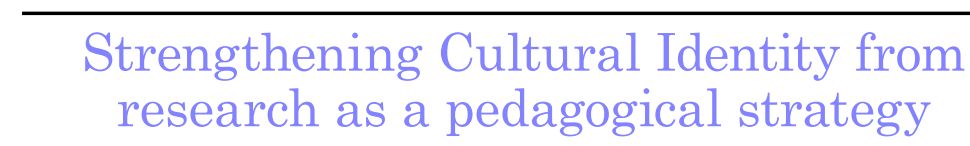

DOI: http://dx.doi.org/10.17981/cultedusoc.9.3.2018.93

Artículo de investigación. Fecha de recepción: 15/06/2018. Fecha de aceptación: 27/11/2018

\author{
Lastenia Contreras-Anaya ${ }^{2}$; \\ Isabel Ariza-Caro; Lastenia Contreras-Anaya; \\ Yeris Castillo-Aguirre y Alexander Polo-Jiménez ${ }^{3}$
}

Institución Educativa Distrital Loma del Bálsamo, sede estación Lleras, Algarrobo (Colombia)

lastenia196907@gmail.com

Para citar este artículo:

Contreras-Anaya, L., María Ariza-Caro, I. Contreras-Anaya, L., Castillo- Aguirre, Y. y Polo-Jiménez, A. (2018). Fortalecimiento de la Identidad Cultural desde la investigación como estrategia Pedagógica. Cultura. Educación y Sociedad 9(3), 791-796. DOI: http://dx.doi. org/10.17981/cultedusoc.9.3.2018.93

\section{Resumen}

El presente artículo muestra resultados derivados del estudio: Fortalecimiento de la Identidad Cultural, desarrollado en la Institución Educativa Distrital Loma del Bálsamo, sede estación Lleras, ubicada en el municipio de Algarrobo del departamento del Magdalena. Evidencia cómo a través de la implementación de la Investigación como Estrategia Pedagógica, apoyada en tecnología de la información y comunicación (TIC); se puede fortalecer la existencia de una diversidad cultural e ideológica que muestran la identidad de una sociedad y de quienes la conforman; especificando la manera cómo los estudiantes de la Institución, se empoderan de los elementos que caracterizan e identifican su idiosincrasia cultural. Señala además como la implementación de la investigación como estrategia pedagógica para el fortalecimiento de la identidad cultural se logra convertir e intervenir un eje transversal del currículo, para que sea abordado desde diversas áreas o contenidos de enseñanza.

Palabras clave: Investigación como estrategia pedagógica, identidad cultural, Tecnología de la comunicación y la información.

\section{Abstract}

This article shows results derived from the study Strengthening Cultural Identity developed at the Loma del Bálsamo District Educational Institution, Lleras station headquarters, located in the municipality of Algarrobo, Department of Magdalena, as evidence through the implementation of Research as a Pedagogical Strategy supported by information and communication technology, ICT; the existence of cultural and ideological diversity that show the identity of a society and its members can be strengthened; specifying the way how from the implementation of research supported by information and communication technology, the students of the Institution, are empowered by the elements that characterize and identify their cultural idiosyncrasy. It also points out how the implementation of research as a Pedagogical Strategy for the Strengthening of Cultural Identity can be converted into a transversal axis of the curriculum so that it can be approached from different teaching areas.

Keywords: Research as a pedagogical strategy, cultural identity, communication and information technology.

1 Este artículo ha sido derivado del Proyecto Fortalecimiento de la Cultura Ciudadana y Democrática en CT+I a través de la IEP apoyada en TIC en el Dpto. del Magdalena.

2 Líder Grupo de investigación "Investigadores Interculturales".

3 Docentes de la institución Educativa Distrital Loma del Bálsamo, sede estación Lleras, en el municipio de Algarrobo (Magdalena).

- The author; licensee Universidad de la Costa - CUC.

Cultura, Educación y Sociedad vol. 9 no. 3, pp. 791-796. Diciembre, 2018

Barranquilla. ISSN 2389-7724 Online 


\section{Introducción}

La identidad cultural se aborda desde diversos aspectos, se considera el factor económico, porque vincula la cultura al mercado y al consumo, esto se manifiesta en las industrias culturales (empresas editoras, casas de música, televisión, cine, etc.). La cultura juega un papel en el desarrollo de la cohesión social, la autoestima, creatividad, memoria histórica, etc. Este es su capital patrimonial, influenciado por políticas públicas orientadas a la conservación, restauración, puesta en valor y uso social de los bienes patrimoniales (Molano, 2007).

El corregimiento de "La Estación Lleras" pertenece territorialmente al Municipio de Algarrobo, ente que tomó su nombre por los amplios cultivos naturales del árbol cuyo fruto se denomina algarroba, fruto que se caracteriza por sus beneficios como alimento forrajero y es utilizado en laboratorios como complemento alimenticio para los seres humanos. Debido a la falta de cultura de los propietarios de estos terrenos, el árbol de algarrobo fue paulatinamente erradicado, de tal manera que los coterráneos lo desconocen, dejando un gran vacío en su historia cultural. Considerando lo anterior, se plantea este proyecto de investigación, enmarcándolo en la línea de Identidad Cultural y Ambiental, trabajar por el rescate de la cobertura vegetal, en el marco de la Investigación como Estrategia Pedagógica (IEP); la cual reconoce a la indagación como una forma de nutrir el proceso de aprendizaje, cambiar las concepciones, realidades y el entorno de los participantes, y con ello desarrollar en loa actores una manera de situarse crítica y éticamente en el mundo cercano, mediato y global, asumiendo responsabilidad por su destino (Mejía y Manjarrez, 2011; Villalba, 2012; Orozco, 2015; Restrepo, 2009).

$\mathrm{Al}$ respecto, se hace necesario trabajar en las escuelas actividades dirigidas a fomentar la identidad cultural, teniendo en cuenta los orígenes de los individuos (Rodríguez, 2015). En ese mismo orden (Molano, 2007), expresa que existen diversas definiciones sobre cultura y todas coinciden en que es lo que le da vida al ser humano: sus tradiciones, fiestas, conocimientos, creencias, costumbres y moral. Esta diversidad de dimensiones y funciones sociales generan un modo de vivir, coexistir, crear riqueza, empleo y equilibrio territorial.

Con frecuencia, se observaba que en las instituciones educativas del país se relegaba el estudio y práctica de los temas relacionados con la identidad cultural y el fortalecimiento de una conciencia nacional. El desafío actual es una formación que incorpore contenidos pertinentes al contexto inmediato y a realidades muy diversas y opuestas a la propia (Morante, 2012).

Maldonado y López, L. (2017), señala que aspectos como la globalización y la revolución tecnológica, podrían llegar a ser considerados como factores limitantes para el rescate y conservación de los valores y la identidad cultural de una región o país, pero dependiendo de su uso y enfoque, pueden llegar a ser favorables para su afianzamiento. Una condición clave para el logro del referido propósito deriva de los procesos de participación ciudadana. Autores como Hernández, Alvarado y Velazco (2013) y De la Concepción (2015) resaltan como necesarios la integración de los individuos a procesos formativos que privilegien una pedagogía centrada en los valores y la convivencia ciudadana.

En este sentido las tecnologías se han convertido en medios que optimizan la difusión masiva, la promoción efectiva de los saberes, costumbres, tradiciones y expresiones artísticas que acercan a nuestros estudiantes al entorno cultural del cual forman parte, pero no se debe dejar de lado que el objetivo de la educación debe ser promover la capacidad reflexiva del niño, de evaluar su realidad y su historia. 
Como lo expone García (2015), en esta época incorporar la tecnología a la educación aporta una serie de beneficios, porque ayudan a mejorar la eficiencia, la productividad en el aula y a aumentar el interés de los niños en las actividades académicas.

De acuerdo a lo anteriormente expuesto, se hace urgente la necesidad de formar ciudadanos capaces de valorar y reconocer su diversidad cultural, y de responder de manera eficiente y eficaz a los retos emergentes a raíz del progreso en todos los ámbitos del ser. Esto es necesario considerarlo, teniendo en cuenta que la identidad cultural es una base para lograr conocer los orígenes de la comunidad y de esta manera afrontar el futuro con carácter y convicción.

Se busca aportar al currículo escolar un conjunto de contenidos que fomenten capacidades y actitudes de identidad cultural, así como al conocimiento de su realidad a partir de su historia, costumbres, creencias, mitos, leyendas, comidas típicas, tradiciones, y los demás escenarios, tradiciones y rasgos propios de las localidades. En este sentido la identidad cultural está asociada a los procesos de desarrollo de las comunidades; tal como señalan Gutiérrez, Carrera, Marín, Narváez y Pérez (2006), deben fortalecerse flujos de relación entre los actores del sistema social que contribuyan a la consolidación de las capacidades y rasgos identitarios de las comunidades.

\section{Metodología}

Núñez y Ravina (2017) citan a los autores Haberland y Dacin los cuales manifiestan que "la investigación en creatividad ha tenido una larga tradición en la psicología y el mercadeo. Mientras que la mayoría de los estudios desde el punto de vista del marketing se orientan hacia la efectividad, los estudios psicológicos se enfocan más en medir la creatividad" (p. 12).

El artículo que se presenta es un producto derivado del estudio para el fortalecimiento de la Identidad Cultural, desde la investigación como estrategia Pedagógica, desarrollado en la Institución Educativa Distrital Loma del Bálsamo, sede estación Lleras; su objetivo principal ha sido fortalecer la existencia de diversidad cultural e ideológica que muestran la identidad de una sociedad y de quienes la conforman; especificando la manera cómo desde la implementación de la investigación apoyada en la tecnología de la información y comunicación; el estudio contempla una metodología con el siguiente orden:

- En su diseño, el estudio utilizó la metodología cualitativa, realizando la descripción del fenómeno a partir de observaciones que adoptan la forma de entrevistas, narraciones, notas de campo, grabaciones, transcripciones de audio, vídeo, registros escritos (Pérez, 2002; Hernández 2014).

- Núñez y Ravina (2017) indican que la creatividad en estos estudios está basada fundamentalmente en tres características fundamentales: originalidad, capacidad de adaptación a la realidad y grado de elaboración. (p.12)

- Se utilizó el modelo de acción participativa, porque esta propuesta metodológica forma parte de una estrategia que involucra a la comunidad en el conocimiento y solución de sus problemas. Se inscribe dentro del campo de las acciones de capacitación que no busca solamente describir los problemas, sino que busca generar conjuntamente con los involucrados en la comunidad, los conocimientos necesarios para definir las acciones adecuadas que estén en la línea del cambio y la transformación de la realidad que requiera su intervención. (Schutter, 1987; Báez, 2009). Siendo esta una metodología de investigación que se aplica a estudios sobre el mejoramiento de la realidad humana, es apropiada en este proyecto, ya que es esta la intención central del estudio. 
- Las unidades de análisis de la población beneficiaria de este proyecto de investigación fueron los estudiantes de la Institución Educativa Loma del Bálsamo, sede Estación Lleras, del municipio de Algarrobo, del departamento del Magdalena. Estos fueron seleccionados de manera intencional puesto que presentan características comunes ante el problema de investigación, en cuanto a la caracterización sociodemográfica, estos habitan en el municipio de Algarrobo, Magdalena y en su totalidad pertenecen a estratos económicos bajos.

- En cuanto a los instrumentos de recolección de información, se utilizó la bitácora o diario de campo, relatorías individuales, institucionales, observación directa, encuestas, información suministrada por los estudiantes y la comunidad.

\section{Resultados}

Se logró realizar una integración curricular en las diferentes áreas del conocimiento, liderado por los docentes investigadores y en apoyo con los docentes en general de la institución. Lo anterior usando como medio investigativo las TIC, implementándolas tanto para investigar, como para difundir la información hallada y construida en el proceso con los estudiantes.

Se dieron espacios institucionales en los cuales se fomentó la apropiación del conocimiento sobre identidad cultural del corregimiento Estación Lleras, asistido por toda la comunidad estudiantil y planta docente de la institución.

\section{Discusión y conclusiones}

En relación con los planteamientos que fundamentan el estudio, y los resultados obtenidos, se presentan las siguientes conclusiones: se requiere la reestructuración curricular y dentro de esta incluir de manera trasversal el proceso del fortalecimiento de la identidad cultural, a través de la implementación de acciones pedagógicas apoyadas en herramientas TIC, que promuevan en la comunidad de estudiantes el aprendizaje y apropiamiento de su origen, historia, ideología, pensamiento sociocultural.

Teniendo en cuenta cómo es concebido el niño en la actualidad, como un conocedor de su postura histórica, el estar consciente de su origen y su trayectoria es fundamental para construir su propia historia; pero también es importante que se prepare para desarrollar conceptos propios de la modernidad e incluso, ser un conocedor de los derechos. Estos conceptos que son tan cotidianos en esta época, que muy pocas personas podrían rechazar, son el ejemplo de un cambio paradigmático en la forma de abordar la formación humana a lo largo de la historia. Este es un desafío que implica una ruptura para la organización de los contenidos de enseñanza y para el diseño de estrategias pedagógicas integrales y contextuales en la actualidad y a futuro (Cruz, 2015).

Para afianzar los procesos cognitivos de los niños que faciliten y potencien el sentido de pertenencia, el conocer su procedencia es importante para desarrollar la identidad y una postura crítica ante los acontecimientos actuales y los que se avecinan.

Para Cruz (2015) el desarrollo de experiencias de aprendizaje que sean creadoras de conciencia de cultura en la niñez, se deben contextualizar; porque es a partir del concepto de socialización, que se entiende el proceso a través del cual el niño adquiere una serie de repertorios sociales, morales y culturales que van a intervenir en la construcción de la identidad y posteriormente van a llevar a contrastar esta identidad construida e interiorizada con nuevos repertorios que se van adquiriendo a lo largo 
de la vida. En el proceso de socialización los niños adquieren los roles, expectativas, creencias y valores que la sociedad provee, brindándole al sujeto una sensación de estabilidad frente al orden social en el que está inmerso. En este orden de ideas, los principales agentes socializadores son la escuela y la familia.

El hilo conductor en la construcción de conceptos que encaminen al sentido de pertenencia, va ligado a la formación en la escuela, a la reflexión sobre posibles situaciones futuras que se avizoran y a la perseverancia de un aprendizaje significativo en favor de la creación de sentido cultural. Fortalecer el proceso de la identidad cultural en estudiantes, es un eje central desde la concepción sobre la diversidad cultural existente en la sociedad que se encuentra inmerso. Esto es lo que se ha denominado consciencia social y cultural.

El niño que construye su identidad a partir del conocimiento producto del proceso de socialización en el contexto escolar, de entrada, se va ubicar en un lugar particular dentro del orden social, con unas características particulares que van a influir en su desarrollo a lo largo de todo su ciclo vital, con conciencia del ambiente que le rodea.

Finalmente, se debe entender que la relación de la niñez y la educación implica tener una aproximación compleja de este fenómeno social que no solo representa una institución como la escuela, sino que implica un sistema de relaciones con los distintos actores y organizaciones sociales, los medios de comunicación, los discursos pedagógicos, políticos, psicológicos, éticos; entre otros.

La educación, entendida como una tarea de todos, basada en la relación no sólo entre educadores y educandos, padres y sociedad; tiene la prioridad de desarrollar conexiones reflexivas sobre las situaciones y características particulares que afectan a los niños, las organizaciones sociales, los medios de comunicación, los discursos sociales e históricos. Estos son escenarios que deben ser considerados como desafíos de transformación en la disposición de la institución educativa a abrirse a la comunidad, a convertirse en promotora de interacciones necesarias para formar integralmente a los ciudadanos en la vida y desde la vida con calidad.

\section{Referencias}

Báez, J. (2009). Investigación cualitativa. Madrid: ESIC Editorial.

Cruz, M. (2015). La niñez en la escuela: Una historia de poder, control y desarrollo. Cultura Educación y Sociedad, 6(1), 127-139.

De la Concepción, A. (2015). Concepciones sobre participación de niñas, niños y adolescentes: Su importancia en la construcción de la convivencia escolar. Cultura Educación y Sociedad, 6(2). Recuperado de https://revistascientificas.cuc.edu.co/culturaeducacionysocie$\mathrm{dad} /$ article/view/840

García, A. (2015). Los beneficios de la Tecnología en la Educación | Artículos. Labrechadigital.org. Recuperado de http://www.labrechadigital.org/labrecha/Articulos/los-beneficios-de-la-tecnologia-en-la-educacion.html

Gutiérrez, C., Carrera, M., Marín, F., Narváez, M. y Pérez, C. Integración de redes académicas para la gestión del desarrollo endógeno regional. Multiciencias 6(3). [en linea]. Recuperado de http://ww w.redalyc.org/articulo. oa?id=90460308

Hernández, J.., Alvarado, P., Lisandro, J. (2013). Participación ciudadana, tecnologías de información- comunicación y opinión pública. TEACS, 5 (11)

Hernández, R., Fernández, C. y Baptista, P.(2014).Metodologíadelainvestigación. México, D.F.: McGraw-Hill Education. 
Maldonado, M. y López, L. (2017). La visión del desarrollo dentro del contexto global y regional. El regionalismo a través de la Alianza del Pacífico y la Asociación Latinoamericana de Integración 20052014. Revista Desafíos, (29-I): 13-48.

Mejía, M. y Manjarrés, M. (2011). La investigación como estrategia pedagógica una apuesta por construir pedagogías críticas en el siglo XXI. Praxis \& Saber, 2(4), 127. http://dx.doi.org/10.19053/22160159. 1127

Molano L. (2007). Identidad cultural un concepto que evoluciona. Revista Opera [en línea]. Recuperado de http://sss.redalyc.org/articulo.oa?id=67500705

Morante, P. (2012). Promoviendo la identidad cultural desde el jardín de infantes. Recuperado de http://www.redem.org/ boletin/boletin150312b.html

Núñez, E. y Ravina, R. (2017). Análisis del nivel de competitividad empresarial en el panorama publicitario colombiano fundamentada en el éxito creativo y la responsabilidad ética y jurídica. JURÍDICAS CUC, 13(1). 9-28. http://dx.doi. org/10.17981/juridcuc.13.1.2017.1

Orozco, M. (2015). Métodos, formas, procedimientos y sistemas de enseñanza en una concepción de la pedagogía como arte según José M. Zamora. Aula, 21(0), 165-176. http://dx.doi.org/10.14201/aula 201521165176
Pérez, G. (2002). Investigación cualitativa. Retos e interrogantes. II técnicas y análisis de datos. Madrid: la Muralla S.A.

Restrepo, B. (2009). Investigación de aula: formas y actores. Bibliotecadigital. udea.edu.co. Recuperado de http://bibliotecadigital.udea.edu.co/bits $\mathrm{t} \mathrm{r}$ e a m/10495/3498/1/ $\mathrm{R}$ estrepoBernardo_2009. Investigaci\%C3\%B3naulasformas.pdf

Rodriguez, O. (2015). La investigación acción y la construcción de la identidad histórico cultural. A la otra orilla. Recuperado de https://alaotraorilla.wordpress.com/2015/05/06/hola-mundo/

Ruggeroni, C. (2002). Los Niños y las Tecnologías de la Información. Psychnology.org. Recuperado de http://www. psychnology.org/File/PSYCHNOLOGY_JOURNAL_1_1_RUGGERONI. pdf

Schutter, A. (1987). Metodo y proceso de la investigación participativa en la capacitación rural. Patzcuaro: CREFAL.

Villalba, A. (2012). Dos paradigmas contrapuestos en la evaluación educativa, dos formas diferentes de concebir al alumno. Aula Universitaria, 1(14). http://dx.doi.org/10.14409/au. v1i14.4123 